Network Working Group

Request for Comments: 2094

H. Harney

Category: Experimental

C. Muckenhirn

SPARTA, InC.

July 1997

\title{
Group Key Management Protocol (GKMP) Architecture
}

Status of this Memo

This memo defines an Experimental Protocol for the Internet community. This memo does not specify an Internet standard of any kind. Discussion and suggestions for improvement are requested. Distribution of this memo is unlimited.

Table of Contents

1. Introduction.............................. 1

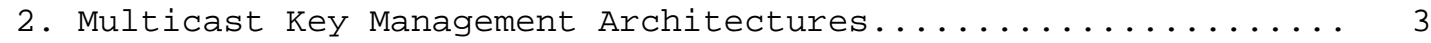

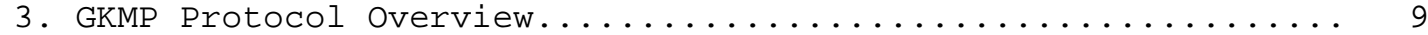

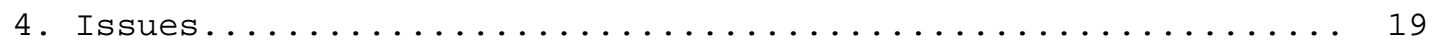

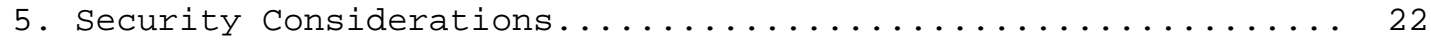

6. Authors' Address............................. 22

Abstract

This specification proposes a protocol to create grouped symmetric keys and distribute them amongst communicating peers. This protocol has the following advantages: 1) virtually invisible to operator, 2) no central key distribution site is needed, 3) only group members have the key, 4) sender or receiver oriented operation, 5) can make use of multicast communications protocols.

1 Introduction

This document describes an architecture for the management of cryptographic keys for multicast communications. We identify the roles and responsibilities of communications system elements in accomplishing multicast key management, define security and functional requirements of each, and provide a detailed introduction to the Group Key Management Protocol (GKMP) which provides the ability to create and distribute keys within arbitrary-sized groups without the intervention of a global/centralized key manager. The GKMP combines techniques developed for creation of pairwise keys with techniques used to distribute keys from a KDC (i.e., symmetric encryption of keys) to distribute symmetric key to a group of hosts. 


\subsection{Multicast Communications Environments}

The work leading to this report was primarily concerned with military command and control and weapons control systems, these systems tend to have top--down, commander--commanded, communications flows. The choice of what parties will be members of a particular communication (a multicast group for example) is at the discretion of the "higher" level party(ies). This "sender-initiated" (assuming the higher-level party is sending) model maps well to broadcast (as in electromagnetic, free-space, transmission) and circuit switched communications media (e.g., video teleconferencing, ATM multicast).

In looking to apply this technology to the Internet, a somewhat different model appears to be at work (at least for some portion of Internet multicast traffic). IDRP and Distance Vector Multicast Routing Protocol (DVMRP) use multicast as a mechanism for parties to relay common information to their peers. Each party both sends and receives information in the multicast channel. As appropriate, a party may choose to leave or join the communication without the express permission of any of the other parties (this begs the question of meta-authorizations which allow the parties to cooperate). More interestingly, the multicast IP model has the receiver telling the network to add it to the distribution for a particular multicast address, whether it exists yet or not, and the transmitter not being consulted as to the addition of the receiver.

Other applications of multicast communications in the Internet, for example NASA Select broadcasts, can be viewed as implementing the sender model since the sender selects the broadcast time, channel, and content, though not the destinations.

It is our intention to provide key management services which support both communications (and implied access control) models and operate in either a circuit switched or packet switched environment.

\subsection{Security for Multicast}

Multicast communications, as with unicast, may require any of the security services defined in ISO 7498, access control, data confidentiality, traffic confidentiality, integrity/data authentication, source authentication, sender and receiver nonrepudiation and service assurance. From the perspective of key management processes, only data confidentiality, data authentication, and source authentication can be supported. The other services, traffic confidentiality, non-repudiation, and service assurance must be provided by the communications protocol, they may rely on cryptographic services but are not guaranteed by them. 


\section{Multicast Key Management Architectures}

\subsection{Current Operations}

There are several electronic mechanisms for generating and distributing symmetric keys to several computers (i.e., communications groups). These techniques, generally, rely on a key distribution center (KDC) to act as a go between in setting up the symmetric key groups. Military systems, such as BLACKER, STUII/BELLFIELD, and EKMS, and commercial systems, such as X9.17 and Kerberos, all operate using dedicated KDCs. A group key request is sent to the KDC via various means (on- or off-line) The KDC acting as an access controller decides whether or not the request is proper (i.e., all members of a group are cleared to receive all the data on a group). The KDC would then call up each individual member of the group and down load the symmetric key. When each member had the key the KDC would notify the requester. Then secure group communication could begin. While this was certainly faster then anything that requires human intervention. It still requires quite a bit of set-up time. Also, a third party, whose primary interest isn't the communication, needs to get involved.

Pairwise keys can be created autonomously by the host on a network by using any number of key generation protocols (FireFly, Diffe-Hellman, RSA). These protocols all rely on cooperative key generation algorithms to create a cryptographic key. These algorithms rely on random information generated by each host. These algorithms also rely on peer review of permissions to ensure that the communication partners are who they claim to be and have authorization to receive the information being transmitted. This peer review process relies on a trusted authority assigning permissions to each host in the network that wants the ability to create these keys. The real beauty of these pairwise key management protocols is that they can be integrated into the communication protocol or the application. This means that the key management becomes relatively invisible to the people in the system.

\subsection{GKMP-Based Operations}

The GKMP described below, delegates the access control, key generation, and distribution functions to the communicating entities themselves rather than relying on a third party (KDC) for these functions. As prelude to actually distributing key, a few things must be assumed (for purposes of this document): there exists a "security manager" responsible for creating and distributing to parties authentic identification and security permission information (The security manager function may be accomplished through a strictly hierarchical system (a la STU-III) or a more ad hoc system of 
cooperating peer "domain managers," the implementation of the certification hierarchy is not addressed in this document.); communicating parties are online for the keys formed and distributed by the GKMP.

\subsubsection{Sender Initiated Operations}

This section describes the basic operational concept for multicast key management for sender initiated multicast support. This model of multicast communications was the basis for our original work on multicast key management. From a security viewpoint the sending application is able to control access to the transmission through both key distribution and communications distribution (not sending the transmission to some addresses).

Identification of Group Key Controller -- The originator of the multicast group creates or obtains a group management certificate from its certification hierarchy. The certificate identifies the holder as responsible for generation and distribution of the group key (Naming standards are not addressed here, the name should reflect the naming structures appropriate for the supported cryptographic service. For example, IP-level encryptors should use naming reflecting "host" identities (IP addresses, or DNS host names), RTP encryptor would use session names). The originator relays the membership list to the Group Key Management (GKM) application.

Group Key Creation -- The GKM application, operating on behalf of the originator, selects one member of the group, contacts it, and creates a Group Key Packet (GKP). A GKP contains the current group traffic encrypting key (GTEK) and future group key encrypting key (GKEK). The GKM application then identifies itself as the group key controller, which the member validates, under cover of the GTEK.

Group Key Packet $(\mathrm{GKP})=[\mathrm{GTEKn}, \mathrm{GKEKn}+1]$

As part of group key packet formation, usage parameters, appropriate for the underlying crypto-system, are selected. Unlike normal parameter negotiation, where common security-level/range, and services are arrived at, the originator's GKM application selects these parameters and the member must comply.

Group Key Distribution -- After creation of the GKP, the group controller contacts each member of the group, creates a Session Key Package (SKP), validates their permissions (check member's certificate against group parameters), and create a Group Rekey 
Package for that member. A SKP contains a session TEK and a session KEK for a particular member. A GRP contains the GKP encrypted in a KEK and signed using the originator's certificate.

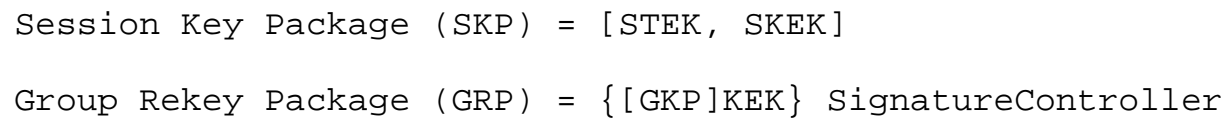

This procedure is fairly complex, but other than for the distribution of site-specific certificates, no centralized key management resources are needed. The only parties to the key management communications are the same parties which will be participating in the group.

\subsubsection{Receiver Initiated Operations}

This section describes key management operational concept for receiver initiated multicast communication support. The receiver initiated model presents some interesting problems from a security view point since the end-participants are not known a priori. Also, in a purely receiver initiated application (such as DVMRP), there is no concept of an "originator" and the participants in the group may be quite dynamic with participants changing on a minute by minute basis.

For secure group communications to take place, all members must obtain the same key. This may be achieved by either using deterministic key generation techniques (using a secret, shared seed) or by making one member of the group responsible for creation of the key. The use of a deterministic key generator presents security problems, particularly regarding loss of the seed (it compromises both past and future traffic). The assignment of a member to the role of key "controller" also presents drawbacks, but these relate to determining which one should be the controller and the need for each member to contact him. The remainder of this discussion will look at how the "controller" concept from above could work in the receiver initiated case.

Selection of Group Key Controller -- A group member will be made responsible for initial group establishment and periodic generation and dissemination of new GRPs. There is no need for the selected controller to be the controller for all time, but at any one time only one controller may be active for each group. Selection of 
controller may be made through a voting system, by a simple default (the first to transmit to the group is the controller), or configuration.

The current controller's identity must be made available to all members, and potential members, for initial group key load and error recovery. The information may be relayed by broacast on a key management "channel," or through a directory service.

Group Key Creation -- The GKP is created and distributed in much the same way as in sender initiated operations. The controller creates a GKP with the first group member to initiate contact. The GKM application then identifies itself as the group key controller, which the member validates, under cover of the GTEK. Parameter negotiation is performed and the first group member is keyed.

Group Key Distribution -- After creation of the GKP, as other members contact the controller, a SKP is created, member permissions are validated and a GRP is loaded to the member.

For widely distributed groups, a form of distributed dissemination may be used. Some number of regional GKM applications are enabled with the ability to validate the permissions of new members and upon validation send to them the current GKP. (Access control is not defined in this document, but it is assumed that both hierarchical and discretionaly (rule-based and identity-based) access control will be supported.) These regional key distributors perform the same functions as the controller, except that they do not create the GKP. This concept can be expanded to the point where all current members are capable of downloading the GKP, and passing on that capability.

Group Rekey -- When the group need rekeying the procedure would be identical to the sender initiated case. The controlling GKM application selects a member, creates a new GKP, creates a new GRP (which is encrypted in the previously distributed next GKEK) and broadcasts it to the group.

\subsection{GKMP Features}

This section highlights areas which we believe the GKMP approach has advantages over the "traditional" KDC based approaches.

\subsubsection{Multicast}

Multicast protocols are a growing area of interest for the Internet. The largest benefit of a multicast protocol is the ability of several receivers to simultaneously get the same transmission. If the transmission is of a sensitive nature, it should be encrypted. This 
means that the all members of the group must share the same encryption key to take benefit of the multicast transmission.

To date the only way of setting up a group of symmetric keys is with the assistance of a centralized key management facility. This facility would act as a key broker creating a distributing key to qualified group members. There are several problems with this centralized concept. These problems give rise to many of the following motivations for creating a distributed key management protocol.

\subsubsection{Increase the autonomy of key groups}

The GKMP proposes to extend the pairwise key paradigm to grouped keys. This protocol can be integrated into the communication protocols or applications and can become invisible to the host's operator. We will use peer review to enforce our security policy.

The GKMP allows any host on a network to create and manage a secure group. Maintenance of these group keys can be performed by the hosts interested in the group. The groups themselves will be relatively autonomous. This simplifies the installation of this technology allowing more host to use secure multicast communications.

\subsubsection{Latency}

Latency refers to the time to set-up or tear down or to re-key a group. In short this corresponds to the length of time it would take to set-up a multicast address.

The GKMP can allow delegation of group creation authority to any host in the network. In essence, when a host needs a group it will have the tools needed to create that group and manage it. Additionally, since the host only needs to create a single group it can concentrate on that particular group.

In the current centralized key distribution approach. The group must be requested from the central site. The central site would process that request in accordance with it's priority and current workload. Latencies would develop if the workload of the central site gets unwieldy or if the communications to the site become overloaded.

\subsubsection{Extendibility}

One of the problems with a centralized key distribution system is the concentration of key management workload at a single site. The process of creating key groups -- key creation, access review, communication to group members takes time and effort. As the number 
of groups on the network grows and the number of group members group. The workload at that central sight quickly reaches capacity.

GKMP should allow a great number of groups to exist on the Internet without overloading any particular host. Delegation of the net wide group creation and management workload places the burden of maintaining groups on the hosts interested in using those groups. Not only is this more efficient, but it places the burden in an appropriate location.

The GKMP distributes the communication requirements to manage groups across the network. Each group manages the group using the same communication resources needed to pass traffic. It is likely that if a communication group can support the traffic of a group, it will be able to support the minimal traffic needed to management the keys for that group.

GKMP provides it's own access control, based on signed netwide permission certificates. This partially disseminates the burden of access control and permission management. A system wide authority must assign the permission certificates, but day to day access control decisions are a GKMP responsibility.

\section{3 .5 Operating expense}

A centralized key distribution site contains, at one time or another, the keys for the net. This is a valuable target for someone to compromise. To protect this site physical and procedural security mechanisms are employed (e.g., guards, fences, intrusion alarms, two person safes, no-alone zones). These mechanisms do not come cheap.

Allowing the hosts to create and manage their keys eliminates the need for an on-line centralized key distribution site. The protocol approach restricts access to the keys to the hosts using them (the minimal set). Since, the encryption mechanisms will have already incurred the cost to be physically secured there is no additional cost levied on the system by the key management system.

\subsubsection{Communication Resources}

Because a centralized site is involved in creating, distributing, rekeying, and providing access control for every group, it is frequently accessed. The communication resources available to this site often become a bottle neck for the groups. Therefore a big pipe is usually installed to this facility. 
The GKMP proposes delegating most of the key creation, distribution, rekey and access control mission to the hosts that need the secure communication. There no longer is a single third party that must be consulted prior to every group key management action. Hence, the communications requirements to manage the keys have shifted to the groups themselves. The need for special high capacity communications has been eliminated.

\subsubsection{Reliability}

Delegating key management responsibility to the groups eliminates the centralized key management site as a single point of failure. The groups that will use the key are responsible for it. If the communications system fails for the key management it is also down for the communications.

The GKMP will attempt to delegate as many functions to the group as possible. There will be some functions which still need to be performed outside of the group (granting of privileges). These functions can still fail. The GKMP will operate on the old set of permissions. These functions need not be in-line. They are performed separate from the key management actions and are not crucial to day-to-day operation.

\section{3 .8 security}

People are the most risky element for security. A distributed protocol eliminates many people from the key distribution chain. This limits "exposure" of the key.

3 GKMP Protocol Overview

\subsection{Supporting functions}

A secure key management protocol needs a number of supporting functions, especially in a military environment. The two major support functions are security management and network group management. In the commercial world a company could provide these support functions.

The issue of Security Management is permission management, in a military environment separation of data occurs along classical classification lines (i.e., TOP SECRET to UNCLASSIFIED). In the commercial world these levels are proprietary or need to know access.

Network group management provides an interface to the communications system and control of network resources. Some entity either a commercial or military system, the host or network operations center, 
must provide the key management protocol with a list of the group members. Also, if the network resources, bandwidth and processing, are considered scarce a management structure must allocate them.

\subsubsection{Security management}

Security management is a role performed for the entire network. It involves netwide issues of permission management, initialization of software, and compromise recovery. The GKMP relies on security management to operate. Refer to figure 1: Security management view.

The GKMP must assume trusted handling of the protocol software prior and during installation. If the GKMP is to use peer to peer access control the system must control the assignment of permissions. These permissions must be monitored and updated as needed. Finally, overview of these permissions must include the maintenance of a Certificate Revocation List.

Secure start-up We need to control the process of loading GKMP software onto a host and initializing it. The protocol needs keys,

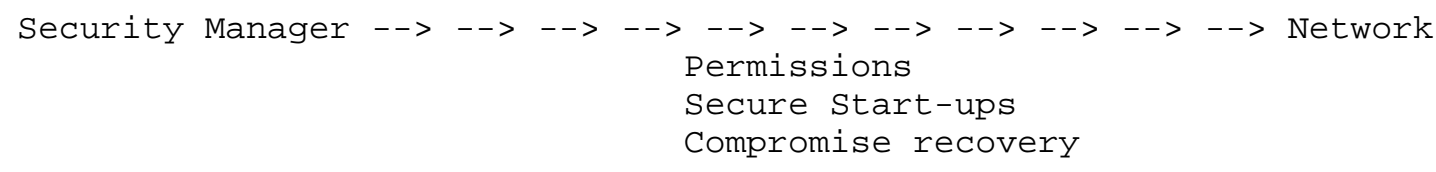

Figure 1: Security Management View

public and private, to operate. It also must have identify

information of the host on whose behalf it will act.

There are some life cycle and security concerns with the software while in transit, stored, distributed, and installed. A one time start-up procedure must verify the identity of the host. Procedural and physical identification techniques will verify the identity of the host (i.e., the Armed Forces Courier Service (ARFCS) accounting, or registered mail). Upon key delivery the security manager logs it's receipt and assumes responsibility for the key.

After proper installation of the software a paper trail verifies the recipient. The computer would initiate an association with the security management function to initialize the protocol software (create a unique public and private key pair for network operation and receive network permissions). This activation process uses keys distributed with the software (good only for initialization) to secure an exchange with the security manager. The host then creates a unique public and private pair and sends the public key to the 
security manager. The security manager creates a credential that uniquely identifies the host and it permissions. This credential is signed by the security management with its private key and can be verified by all net members with the public key.

Permission management Each host on the network is given a permissions certificate signed by the security management which uniquely identify that host and identifies the access permissions it is allowed. These permission certificates are used by the network hosts to assign permissions to other hosts.

This process assigns permissions to equipment or human beings in accordance with their duties. This process involves security clearances and human judgment therefore it is outside the scope of this protocol.

The security management function, especially in military operations, would be responsible for managing permissions and classifications at each host. In the commercial world, permission management corresponds to projects or duties.

Compromise recovery management If a group member is found compromised, the protocol must facilitate the exclusion of the compromised member and return to secure operations. The security management function will provide control of compromise recovery.

Usually, physical inspections or accounting techniques find compromises. These separate systems report the compromise to the key management system. We must assume the loss of all key resident at that host. The security management function will rescind the permission allocated to this compromised host. We create a list of all know compromised hosts and distribution that list across the network. Each host is then responsible for reviewing the propriety of each association and enforcing access control to data.

\subsubsection{Group management}

The group manager interacts with other management functions in the network to provide the GKMP with group membership lists and group relevant commands. The GKMP deals strictly with cryptographic key. It relies on external communication and network management services to supply network related information. Primarily, it relies on the network management service to provide it with the addresses of group members (if the group is sender initiated). 
The GKMP allows an external entity to determine the controller of a group. The controller of the group should be able to handle the additional processing and communication requirements associated with the role. If this is not a necessary function given the implementation, this assignment of controller duties can be set to some automated default. However, even if defaulted some external management entity determines how the role of controller is allocated.

The group manager can receive group progress reports from the group controller. The GKMP provides a service for the network. It makes sense that someone in the network is interested in the progress of this service. The GKMP can provide progress reports. It is up to the network management to determine the manner and recipient of the reports. Reference figure 2: Network manager interaction.

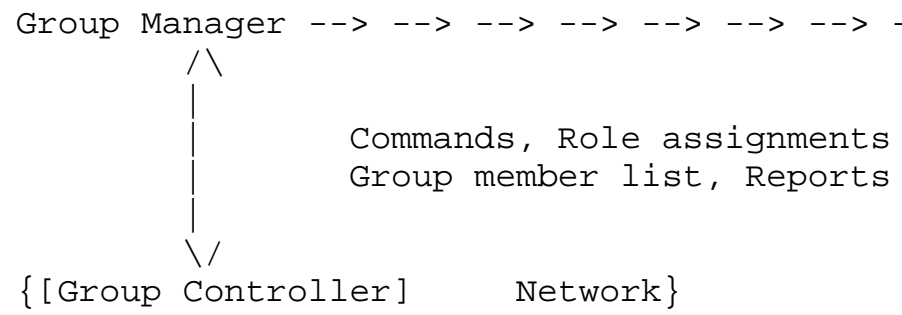

Figure 2: Network Manager Interaction

Group to member mapping When the GKMP is implemented in sender initiated group establishment mode, a list of group member addresses must be provided as part of the group establishment command. The GKMP will use these addresses to contact the group members and create the group.

The creation of groups involves the assignment of a group address, update of router databases, and distribution of this group address to the group members. This is a classic function of network management. The GKMP group controller would be another recipient of this information.

Protocol role allocation The Group Management Protocol assigns roles to members of a particular group. These roles are binary one is either the control over the group or a member of a group. Some external entity will allocate the identity of the group controller and group receiver. This is a desirable aspect because some computers are more capable (i.e., central site, great deal of process power available to control a group). We allow some external entity to allocate these roles to individual group members, this is important in the military application do to the fact that in a 
commercial application the allocating authority and group controller may very well always be the same.

Group key progress reporting The Group Key Management Protocol has to be able to report to somebody. If we create a group, we should report it to group requester. Contrarily if we are not able to

Network $=\{$ [(Group 1 controller) Group 1 members],
[(Group 2 controller) Group 2 members], [(Group 3 controller) Group 3 members], \}

Figure 3: Distributed Group Management

create a group we should report that especially since failure to create a group at least as a first study will highly correlate with a failure of the underlying communications. The Group Key Management Protocol does not have an ability to fix the underlying communications so the communication management function must deal with these failures.

\subsection{Protocol Roles}

Creation and distribution of grouped key require assignment of roles. These identify what functions the individual hosts perform in the protocol. The two primary roles are those of controller and receiver. The controller initiates the creation of the key, forms the key distribution messages, and collects acknowledgment of key receipt from the receivers. The receivers wait for a distribution message, decrypt, validate, and acknowledge the receipt of new key.

One of the essential concepts behind the GKMP is delegation of group control. Since each host in the network has the capability to act as a group controller, the processing and communication requirements of controlling the groups in the network can be distributed equitably throughout the network. This avoids potential single points of failure, communication congestion, and processor overloading. Refer to figure 3: Distributed group management.

\subsubsection{Group controller}

The group controller is the a group member with authority to perform critical protocol actions (i.e., create key, distribute key, create group rekey messages, and report on the progress of these actions). All group members have the capability to be a group controller and could assume this duty upon assignment. 
The group controller helps the cryptographic group reach and maintain key synchronization. A group must operate on the same symmetric cryptographic key. If part of the group loses or inappropriately changes it's key, it will not be able to send or receive data to another host operating on the correct key. Therefor, it is important that those operations that create or change key are unambiguous and controlled (i.e., it would not be appropriate for multiple hosts to try to rekey a net simultaneously).

\subsubsection{Group receiver}

Simply stated a group receiver is any group member who is not acting as the controller. The group receivers will: assist the controller in creating key, validate the controller authorization to perform actions, accept key from the controller, request key from the controller, maintain local CRL lists, perform peer review of key management actions, and manage local key.

\subsection{Scenarios}

\subsubsection{Group establishment}

The protocol to establish a group of host that share a cryptographic key must create a high quality key, verify that all intended recipients have permission to join the group, distribute the key to all qualified members, and report on the progress. This process consists of two phases: creation of the key and distribution of the key. Refer to figure 4: Group Establishment.

The group establishment process is proceeds in the following manner. First, a "create group" command is issued to the group commander. The group controller validates the command to ensure it came from an authorized commander and the group is within the controller's permission range. Next, the controller creates a key. Then that key is passed to the group members, after they pass the peer to peer review process. 


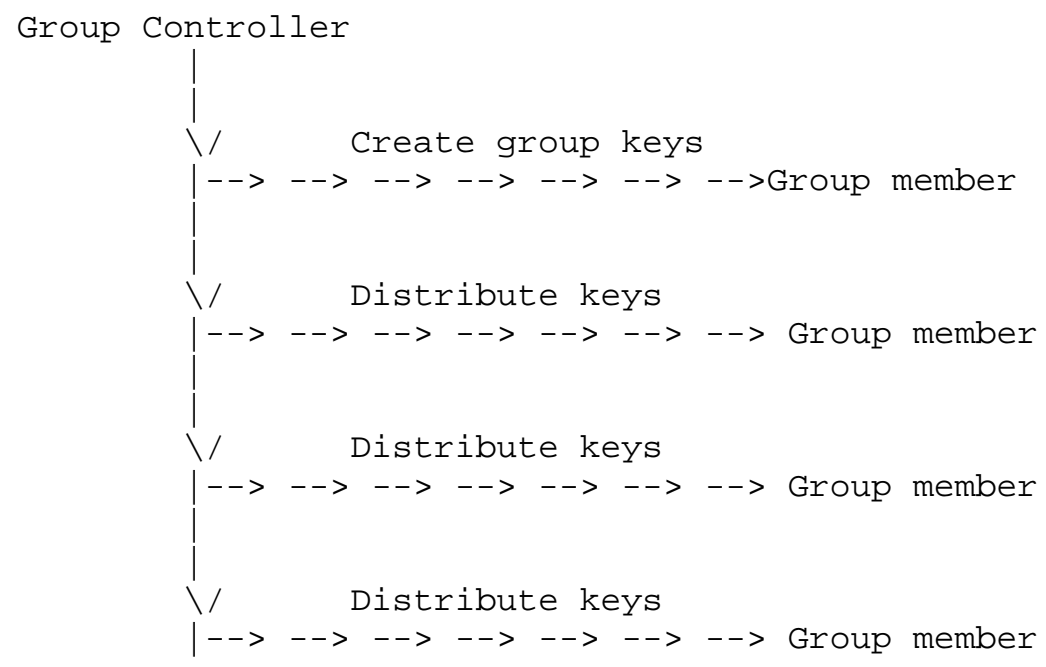

\section{Figure 4: Group Establishment}

Validate command The create group command is signed by the group commander ( they may be the same device). This signature should be asymmetric in nature. The public key to validate this command can be sent with the command itself, if the public bound to the identity of the commander.

The group controller receives the command. It verifies that the signature, thereby ensuring the message was sent by the claimed source and the message has not been modified in transit.

Creation of group keys The controller initiates the creation of two keys for use in the group. The creation of a cryptographic key requires that the key be sufficiently random. Randomizers, capable of creating high grade cryptographic key, tend to be hardware based and are not likely to be practical for this protocol. There are several established key creation protocols based in software (e.g., Diffe-Hellman, FireFly, RSA). All these software based algorithms involve two hosts cooperating to create a cryptographic key. These software algorithms are more appropriate for this protocol.

Also important, in the creation of these keys, is verification of the authorization of the key creation partner. Authorization to posses the keys include permissions that equal or exceed the group traffic and identity verification. 
Distribution of group keys The controller distributes the group keys to the net members. The controller must verify the identity and permissions of each member prior to the key being distributed.

Group Controller $-->-->-->-->-->-->\{$ Group (group member 1-n) $\}$

Figure 5: Group Rekey

Likewise, the net member must verify the controller's identity, authorization to perform this action, and permissions.

The key being distributed is the same level as the data that it will encrypt. Hence, we must encrypt the key during distribution. If no suitable key exists between the controller and member, a new key must be created. This new key is cooperatively created between the controller and net member in a similar manner as the net keys.

The controller creates a message for encryption in the key held between the controller and member. This message will include key management information and the keys.

\subsubsection{Group rekey}

Cryptographic key has a life span. New key must replace "old" key prior to the end of its cryptographic life. This process is rekey.

Rekey has the advantage of using an existing cryptographic association to distribute key. Also, there is no requirement to verify the identity and authorization for the other members. Identify and authorization are assumed.

A group rekey consists of two stages. First the Group Controller creates new group keys. Second these "new" keys are sent to the Group Members in a multicast message. Refer to figure 5: Group Rekey •

Creation of group keys The controller of the rekey will create the new keys in exactly the same manner as used during group establishment. 
Distribution of group keys The GKMP creates a message for the group address. This message uses one of the keys distributed during group establishment to encrypt the new keys. It also contains an authorization token identifying the controller as the rekey agent and new management data. All members of the group using a multicast protocol (if one exists) accept this message.

The message which rekeys the group encrypts the new keys in the existing KEK. Since all group members possess the KEK the entire group can decrypt this message.

The token authorizing the group controller to perform this rekey is also included. This token is asymmetrically signed by the group commander. It uniquely identifies the group controller's authority to rekey this group. It also identifies the group the level of traffic and rekey interval.

\subsubsection{Deletion}

It is desirable to be able to delete group members for either administrative purposes or security reasons. Administrative deletion is the deletion of a trusted group member. It is possible to confirm the deletion of trusted group members. Security relevant deletion is the deletion of an untrusted member. It assumes that the member is ignore all deletion commands.

Administrative delete Administrative deletion removes the group keys from trusted group members. This deletion consists of two messages the first sends a command to the group encrypted in the groups TEK. The command essentially says: acknowledge receipt and then delete group keys. This command is signed by the group controller to prevent unauthorized deletions.

The acknowledgment message is also encrypted under the group TEK and is sent to acknowledge receipt of the command. We could acknowledge accomplishment of the command if the net is willing to accept the burden of creating pairwise keys between the exiting group members and the group controller.

Compromise recovery Compromise recovery is the deletion of untrusted group members. This actually involves the creation of an entirely new group, without the untrusted member. Once the new group is created, net operations can be shifted to the new group, effectively denying the untrusted member access to the data. 
There is always a trade-off between security and continued net operations when a member is found to be compromised. The security first position states that if a member is compromised, the group must be destroyed and then a new secure group created. However, operational concerns sometimes out weigh the security concerns. The operational position is that the group will continue to operate with the compromised member and will shift to a new secure group when it becomes available.

The GKMP does not mandate either position. However, the speed and flexibility of the GKMP does allow a new secure group to be created quickly. Thereby, restricting the potential damage done by a compromised member.

Once a member is found to be compromised, that members certificate is added to a Certificate Revocation List (CRL). The CRL is an asymmetrically signed piece of data, signed by a security manager. The list is made up of compromised resource ID's, a version of the CRL, and perhaps an identifier of the security manager. The CRL is accessed every time a new key is negotiated. If one of the key creators is on the CRL the key is destroyed and interaction terminated.

The idea behind a CRL is each host would keep records of all open associations and compromised resources. The host would then make sure that it does not have and will not create a secure association open with anyone who is on the CRL. The CRL concept of becomes more complicated in the case of groups. This is because it is not necessary for every member in the group to know who the other group members are. Hence, a group member does not have sufficient information to identify compromised group associations. The GKMP proposes that the group controllers be responsible for reviewing the CRL and taking appropriate actions should a group member be compromised.

Another issue with CRLs is the speed that they can be distributed across a network. Every time a key is created the cooperating hosts exchange the version number of their current CRL. If the versions do not match. The most current version is passed to the host with the old version. Hence, CRLs propagate when keys are created. If this is infrequently and there is a single CRL insertion point, the list may take a few days to move across the net. The GKMP allows a speedier distribution of the CRL.

The GKMP delegates control of groups to specific group controllers (a subset of the network). These controllers are responsible for maintaining the security of the group. If quicker distribution of the CRL were desired, the CRL generator ( security management 
function could seed the CRL at these controllers. Controllers are points of key management activity and are logical CRL staging areas.

4 Issues

What are the unresolved issues with this protocol?

\subsection{Access Control}

One interesting issue with a grouped key protocol is access control. This is because we are moving away from having humans in the loop or having a central authority to check the propriety of the group.

The group protocol must police itself. It must ensure that each member of a group meets the classic military access control policy ( i.e., a group member's classification level must be higher or equal to the classification of the group that it's in).

Is allocation of permissions by a higher authority sufficient to provide access control? Or is a more discretionary mechanism necessary?

\subsection{MLS}

A GKMP must be capable of operating in a multi-level secure environment. The integration of a key management protocol capable of creating keys of several different classifications with an operating system capable of operating with multiple classifications in nontrivial.

Classified label standards needed to be incorporated. The classification labels used by the key management protocol should coincide with the labels used by the MLS operating system. These interoperability issues need to be addressed.

\subsection{Error Conditions}

A group protocol is more complex than a pairwise protocol hence there are more possible error conditions. In a pairwise protocol you have two parties; they must communicate between themselves. It is relatively simple to define an take care of all the potential error conditions. 
One assumption with any group protocol is the underlying internet is, to some degree, always broken. The protocol designer has to assume that messages will be delayed or destroyed in transit, all member will not receive all multicast messages, and acknowledgment of actions may not be delivered. This assumption is important if a protocol uses multicast functions to speed-up actions.

The protocol must provide recovery mechanisms to allow group members to recover from loss of messages. It must recover in a way that is transparent to the host and underlying communications network.

For example, there is an issue whether or not we can create an application layer acknowledgment of multi-cast actions. The issue deals with the required bandwidth that acknowledgment would take up. It may be much more friendly to the underlying communications systems to have each member identify potential errors and correct them in a pairwise manner. The task of handling error conditions in a key management protocol is double difficult because many error conditions can be induced error condition (invoked by a third party trying to break the security of that system) to retrieve there key that is in transit or to block the successful dissemination of a key thereby attacking the system security mechanism.

4.4 Commercial vs. Military

Commercial and military key management differ in many ways. Commercial Key management protocols tend to emphasize interoperability, freedom of action, and performance. Military systems tend to emphasize security and control of operations.

There will be a difference in cryptographic algorithms. The military protocol would certainly use high grade encryption because of protecting classified information. The commercial system would probably using algorithms. and techniques certified for unclassified communication systems. The main difference is in the algorithms length and type.

A military protocol would require more management and structure than a commercial one. The military has always adopted a hierarchical communication structure and the commercial system, especially if you look at the internet, work mainly by anarchist style.

\subsubsection{Algorithm Type}

Another difference between military and commercial key management is the type of cryptographic algorithms. The commercial world uses encryption algorithms like DES and in the future Skipjack. The military uses other cryptographic algorithms that differ in key 
length and have more restrictions. An example of this would be the identification of ACCORDION, as a military key encryption algorithm as used in the EKMS program run by NSA.

Any experiments with a grouped key management protocol must consider the differences between military and commercial algorithms. The commercial algorithms tend to be quicker to implement, run faster, involve less processing time, and allows an unclassified experiment. However, we must be careful not paint an unrealistic picture of the performance of the protocol based on these commercial algorithms. A military algorithm tends to be more cumbersome to process, slow to process, require more bandwidth, a lot of unpleasant characteristics from the commercial stand point, but allow for a higher grade of cryptographic security. One way of dealing with the disparity between algorithms is to use the commercial cryptographic algorithms and leave the fields the size used by a comparative DOD cryptographic algorithms and insert delays to simulate DOD algorithm processing times.

\subsubsection{Management Philosophy}

Management for a military network is far more structured than a commercial network. A military network would restrict the creation of network groups, the rekeying of those groups, and access to the data contained in those groups. In contrast the commercial world would enable any member in the network to create a group and allow any other member of the net to join that group.

The group Key Management Protocol must allow for both these architectures i.e., all for very structure command control hierarchy and another free form group creation.

\subsection{Receiver Initiated Operations}

How do they actually work, what are the performance trades, experimentation needed.

Who is the group leader?

How do we elect a new leader?

Will multiple leaders be created?

Will rule based access control allow fine enough access disgression? 
Methods for distributed GKP/GRP dissemination need to be examined. This includes:

- resolving group identification issues, such as how to notify potential members of membership requirements without compromising any security-relevant information about the group;

- approaches for rapidly identifying GKP/GRP sources must be developed, such as a "Key ARP" whereby a new member broadcasts into the group a request for key service and existing members resolve which will provide service; and,

- Security effects of distributing access control decisions must also be reviewed.

5 Security Considerations

This document, in entirety, concerns security.

6 Addresses of Authors

Hugh Harney

SPARTA, Inc.

Secure Systems Engineering Division

9861 Broken Land Parkway, Suite 300

Columbia, MD 21046-1170

United States

telephone: +1410 3819400 (ext. 203)

electronic mail: hhecolumbia.sparta.com

Carl Muckenhirn

SPARTA, Inc.

Secure Systems Engineering Division

9861 Broken Land Parkway, Suite 300

Columbia, MD 21046-1170

United States

telephone: +14103819400 (ext. 208)

electronic mail: cfmecolumbia.sparta.com 